\title{
ESTRATEGIAS DE COLABORACIÓN 2.0 PARA LA TRANSFERENCIA DE CONOCIMIENTO
}

\author{
Lillyana María Giraldo Marín* \\ Luis Joyanes Aguilar* \\ Víctor Hugo Medina García**
}

Recibido: 04/02/2013

Aprobado: 25/10/2013

\section{RESUMEN}

En este artículo de investigación se analiza la colaboración 2.0, donde los empleados pueden participar e interactuar de manera individual y colectiva. La Enterprise 2.0 pone en práctica nuevos modelos de colaboración en la organización los cuales permiten a los empleados establecer escenarios donde pueden mejorar o crear nuevos productos y servicios desde estrategias colaborativas que los conduzcan al logro de un objetivo colectivo. Por lo tanto, se realiza una revisión del concepto de colaboración 2.0 en la organización y como resultado se definen algunas estrategias de colaboración 2.0 en las organizaciones que usan intensamente las tecnologías y los servicios de la Web 2.0 como apoyo a sus estrategias de negocio.

Palabras clave: colaboración 2.0, Enterprise 2.0, organización 2.0, estrategias de colaboración, web 2.0.

DEA en el Doctorado de Ingeniería Informática de la Universidad Pontificia de Salamanca. Maestría en Educación de la Universidad de Manizales-CINDE, Especialista en Gerencia de Información Universidad de Medellín, Ingeniera de Sistemas de la Universidad de San Buenaventura- Medellín. Profesora asociada programa de Ingeniería de Sistemas en la Universidad de Medellín. Carrera: 87 No. $30-65$ Medellín-Colombia. Teléfono: 3405392 o3147288152.1mgiraldo@udem.edu.co

* Doctor en Ingeniería Informática de la Universidad Pontificia de Salamanca, profesor catedrático de la Escuela Superior de Ingeniería y Arquitectura de la Universidad Pontificia de Salamanca. Paseo Juan XXIII, 3 - 28040 - Madrid-España. Teléfono: +34 900 101829. joyanes@ gmail.com

*** Doctor en Informática de la Universidad Pontificia de Salamanca. Maestría en Informática de la Universidad Politécnica de Madrid. Es pecialización en Marketing de la Universidad del Rosario. Ingeniero de Sistemas de la Universidad Distrital. Docente del Doctorado en Ingeniería, Maestría en Ciencias de la Información y las Comunicación y del programa de Ingeniería de Sistemas de la Universidad Distrital “Francisco José de Caldas". Carrera 7 No 40-53 Piso 4, Teléfono 3133338842, Bogotá. vmedina@udistrital.edu.co 


\section{COLLABORATION 2.0 STRATEGIES FOR TRANSFER OF KNOWLEDGE}

\section{ABSTRACT}

This research article analyzes the Collaboration 2.0, where employees can participate and interact either individually or collectively. Enterprise 2.0 puts into practice new collaboration models within an organization; these models allow employees to establish scenarios where they can improve or create new products and services from collaborative strategies which may drive them to achieve a collective objective. Accordingly, a review of the concept of Collaboration 2.0 within the organization is performed, and some Collaboration 2.0 strategies (which intensively use technologies and services of the Web 2.0 as support of their business strategies) within the organization are defined.

Key words: Collaboration 2.0; Enterprise 2.0; Organization 2.0; collaboration strategies; Web 2.0. 


\section{INTRODUCCIÓN}

La organización 2.0 es una estructura en constante cambio y donde las personas participan, colaboran, son abiertas, con visión global, y sin miedo al cambio, estos empleados son los nuevos protagonistas y agentes capaces de dar respuestas a los nuevos desafíos. Como resultado de estas nuevas dinámicas surge la nueva organización, donde pueden participar agentes desconocidos hasta el momento, que deberá aprender a reinventarse de forma permanente y en clara sintonía con tales cambios. Y donde el éxito de la empresa, su fuerza, energía y permanencia no está en sus fuentes de producción, sino en el conocimiento, talento y competencias de sus trabajadores, actuando colectivamente, adaptados a las nuevas tecnologías y sin miedo a lo nuevo e inesperado [1].

Obviamente, ante esta coyuntura, los modelos o formas de gestión tradicionales no pueden dar respuesta a estas nuevas demandas y por lo tanto deben ser remplazados por otros que presten su atención de manera prioritaria en las redes que conectan la empresa y que, además, son los facilitadores del uso pleno de las capacidades y conocimientos de los trabajadores que usan intensamente las tecnologías y los servicios de la Web 2.0 [1], como soporte a estas nuevas demandas de la organización 2.0 .

Al respecto, los autores Frost y Sullivan (2009) establecen que todos los días más y más personas incrementan su trabajo en los espacios virtuales, trabajando desde diferentes ubicaciones, separados por grandes distancias de sus compañeros de trabajo, socios y clientes. Algunos trabajan desde oficinas tradicionales, mientras otros trabajan desde sus casas, oficinas remotas, hoteles, aeropuertos, etc. Es por esto que los empleados, clientes, grupos, socios, entre otros necesitan estar conectados y listos para trabajar juntos, es decir, en ambientes colaborativos, sin tener que viajar, sin tener que encontrarse de manera presencial y lograrlo sin perder la productividad en sus puestos de trabajo y, para ello, las empresas están recurriendo a una variedad de tecnologías de colaboración basadas en IP, tales como: audio, video, conferencia Web, gestores de contenido, mensajería con video inmerso, blogs, wikis, postcast, etc. Estas herramientas, servicios y tecnologías buscan aumentan la experiencia colaborativa de las personas, potencian su productividad y mejoran el flujo de conocimiento del proceso empresarial intra e interorganizacional [2].

\section{FUNDAMENTOS DE LA COLABORACIÓN 2.0}

Si bien la colaboración es un concepto que puede tener varias definiciones según su contexto de aplicación, en los siguientes apartados veremos el concepto de colaboración como escenario que se construye a partir del uso y aplicación de los principios, servicios y tecnologías de la Web 2.0, como soporte a la transferencia de conocimiento intra e interorganizacional.

La colaboración, es definida por los autores [3], en su texto Collaboration 2.0 Technology and Best Practices for Successful Collaboration in a Web 2.0 World, como "la adopción de un conjunto de herramientas y modos de interacción basada en la Web 2.0".

De igual modo, [4] especifica que "[...] la «generación Y» ha desarrollado un nuevo tipo de práctica intensiva de colaboración a través del uso de las tecnologías de la Web 2.0. La colaboración 2.0 es una de las principales actividades de la Empresa 2.0".

De otro lado, los autores Coleman y Levine (2008) definen que "la colaboración 2.0 es una colaboración emergente (no planeada e informal), abierta (indiferente a las fronteras organizacionales) y masiva (implica multitudes)”.

Ampliando las anteriores definiciones sobre como el uso exponencial de las tecnologías y servicios de la Web 2.0 ha mejorado los niveles de colaboración en las organizaciones, los autores [7] definen que: "Un gran número de empresas han 
adoptado exitosamente la Empresa 2.0 para las diferentes actividades de colaboración. Shuen (2008); McAfee (2008); Sari, Schaffers, Kristensen, Loh y Slagter (2008) utilizaron el concepto Enterprise 2.0 para hacer referencia a cómo las tecnologías de la Web 2.0 se pueden personalizar para mejorar la forma de comunicarse en un entorno empresarial y, por ende, mejorar la colaboración”.

En resumen estas definiciones apuntan a que la colaboración 2.0 es un proceso donde dos o más individuos, grupos u organizaciones trabajan juntos, con el fin de realizar una tarea o alcanzar una meta. Se trata de una serie de interacciones, comunicaciones, reflexiones y otras actividades como la búsqueda de información, el hacer preguntas, y recoger las respuestas, generar ideas y resolver problemas. La colaboración es un proceso donde las personas unen sus esfuerzos y trabajo para lograr mejorar o innovar sobre el diseño de productos y servicios, desarrollar una estrategia, trabajar con socios de negocios, resolver problemas y aprovechar las oportunidades.

La colaboración en el contexto de este artículo se presenta como las plataformas tecnológicas que van a soportar el proceso de transferencia de conocimiento 2.0, en esencia estas plataformas tecnológicas están conformadas por un gran número de herramientas y tecnologías electrónicas. En relación con lo dicho, los autores Frost y Sullivan (2009); Turban et al. (2010) y Boughzala (2012) manifiestan que la colaboración cuenta con un gran número de herramientas electrónicas que la soportan. La colaboración requiere que los individuos trabajen juntos de manera coordinada, para lograr un objetivo común. Así, la colaboración es trabajar con otros para lograr objetivos compartidos y explícitos.

La colaboración 2.0 se enfoca en realizar tareas o misiones y, por lo general, se lleva a cabo en una empresa y entre empresas, los empleados demandan estar conectados y usan los medios para lograrlo. Por tanto, la colaboración destaca dos aspectos importantes: primero, la sincronía y la asincronía de las tecnologías, es decir, el tiempo y, segundo, el espacio, ya que estas tecnologías permiten la comunicación entre diferentes personas sin importar su ubicación geográfica [8].

Una de las componentes más importantes de las plataformas colaborativas basadas en la Web 2.0 es la motivación de los participantes a realizar contribuciones que generen valor, para lograr este objetivo los directores de TI de las organizaciones 2.0 deberán reinventar las estrategias hasta ahora desarrolladas con las tecnologías tradicionales y definir estrategias que permitan a los individuos, los grupos, las redes y la organización participar e interactuar en un escenario de colaboración abierto y disponible. En el siguiente apartado se definen algunos principios de las plataformas colaborativas 2.0:

\subsection{Principios de la Colaboración 2.0}

El proceso de colaboración deberá proporcionar a los distintos actores del proceso de transferencia de conocimiento 2.0 las características que se describirán a continuación; de tal manera que estos se sientan motivados a permanecer en el espacio virtual, donde se les ofrezcan las condiciones necesarias que permitan la interacción y la participación; asimismo, donde puedan conformar redes de cooperación basadas en relaciones de confianza que permita el establecimiento de vínculos fuertes y de largo plazo, estos son:

- La transparencia entre los usuarios, esta se puede dar a través del uso de las tecnologías o servicios de la Web 2.0, tales como: blogs, microbloggings (Twitter), gestores de contenidos, wikis, entre otras. La transparencia entre usuarios motiva a los participantes del proceso de transferencia de conocimiento 2.0 a establecer relaciones basadas en la confianza, lo que se traduce en el establecimiento de vínculos fuertes entre los usuarios de las redes sociales y en acuerdos de cooperación basados en la transparencia. Esta transparencia es posible 
gracias a las tecnologías de la web 2.0, las cuales permiten identificar, entre otras características del participante: su perfil, el valor de la contribución, la actualidad de la contribución y el impacto de la contribución en la redes sociales internas y externas de las cuales haga parte el participante.

- La participación espontánea o natural de los usuarios, la cual se puede dar a través de las tecnologías y servicios de la Web 2.0 tales como: blogs, comunidades en línea y redes sociales, videoconferencias, RSS, mensajería instantánea, wikis, postcats, comunidades, redes sociales etc. En este sentido, [9] expresa que en la Web 2.0 hay una "arquitectura implícita de participación, una ética de cooperación inherente, en la que el servicio actúa sobre todo como intermediario inteligente, conectando los extremos entre sí y aprovechando las posibilidades que ofrecen los propios usuarios". Esta característica es muy importante para el proceso de transferencia de conocimiento 2.0, ya que la participación espontánea de los actores del proceso de transferencia de conocimiento permite la creación o el refinamiento de las redes sociales existentes en la organización, como estructuras de cooperación a través de las cuales se lleva a cabo la transferencia de conocimiento.

- La confianza en el grupo: la confianza es un componente determinante para el proceso de transferencia de conocimiento 2.0. Esta se logra cuando algún usuario o contacto hace un aporte individual y deja que la comunidad decida sobre la importancia del mismo. De esta forma, la confianza se convierte en un factor fundamental para motivar la participación de los individuos, grupos, proveedores y clientes. Además, es una característica muy importante en la exteriorización del conocimiento, pues los individuos proponen sus ideas a los grupos o redes intra o interorganizacionales y son los grupos los que, en consenso, definen la validez del concepto para luego continuar con la fase de combinación del conocimiento.

- La disponibilidad de información y conocimiento: Hace referencia al acceso a diferentes fuentes de almacenamiento de información y conocimiento como son: repositorios (bases de datos), programas como SharePoint o wikis, comunidades, sitios Web, blogs, entre otros. La disponibilidad de la información motiva a la participación de los actores de la transferencia de conocimiento (empleados, grupos o redes intra o interorganizacionales), porque pone a su disposición bases de conocimientos individuales y organizacionales actualizadas. Esta característica es muy importante en la fase de combinación del conocimiento.

- La cooperación: los escenarios de colaboración sirven como soporte a las redes sociales, como estructuras a través de las cuales avanza la transferencia de conocimiento; por tal motivo, es importante que la organización tenga una cultura orientada a la colaboración que permita la conformación espontánea de redes sociales y donde sus miembros estén dispuestos a cooperar y a compartir lo que saben.

- La interactividad: La posibilidad que brindan las tecnologías y servicios de Web 2.0 para que las personas interactúen y participen, es uno de los muchos beneficios que su uso en un contexto organizacional generan. La interactividad posibilita la participación y la participación permite la creación y la mejora de servicios o productos en la organización.

De acuerdo a lo anterior, y como ejemplo del impacto que ha tenido la colaboración en algunas empresas del mundo, se presentan algunos de los resultados del estudio Meetings Around the World II: Charting the Course of Advanced Collaboration, llevado a cabo en tres continentes (Asia, América y Europa) y en más de 3000 empresas y realizado 
por los autores Frost y Sullivan (2009) en el cual mencionan que "dentro las compañías que han desarrollado herramientas colaborativas el 72\% creen que han tenido mejor rendimiento, comparado con un $46 \%$ que no las han implementado. La diferencia entre las que desarrollaron una estrategia de colaboración en ventas y las que no es de $76 \%$ vs. $50 \%$ de crecimiento en ventas respectivamente y un crecimiento en beneficios del $71 \%$ y $45 \%$ respectivamente".

Por otra parte, entre las múltiples aplicaciones que tienen los modelos de colaboración 2.0 en la actualidad, una de ellas es apoyar escenarios que favorezcan la transferencia de conocimiento entre individuos, redes, en la organización y entre organizaciones de manera más consciente, donde todas las contribuciones queden registradas y se puedan valorar y apreciar como activos de conocimiento. La colaboración 2.0 permite no solo valorar la transferencia de conocimiento como tal, sino también otros componentes que surgen de la relación que se establece entre emisores y receptores en un escenario de colaboración soportado por acuerdos de cooperación, estos otros activos de conocimiento pueden ser: identificación de expertos, actualizar contactos, establecer nuevas relaciones, identificar flujos de conocimiento intra e interorganizacionales, actualización de bases de conocimiento individuales y organizacionales, mejores prácticas sobre estrategias de colaboración, participación y construcción social de conocimiento con empleados, clientes, proveedores, socios, etc.

Para que estas dinámicas de colaboración se logren, la organización debe facilitar la construcción de redes donde las personas puedan interactuar libremente, registrar sus contribuciones y colaborar en la organización y por fuera de esta. En ese sentido, los autores Turban et al. (2010) proponen que "la colaboración 2.0 ofrece la implementación de la Web 2.0 basada en herramientas del software social y servicios, tales como wikis, blogs, foros, canales RSS, encuestas de opinión, chats de la comunidad y las redes sociales, para facilitar la colaboración en la organización”.

\section{ARQUITECTURA DE LA COLABORACIÓN 2.0}

Con el objetivo de tener un mejor entendimiento de la colaboración 2.0 y complementando la definición sobre la misma, Turban et al. (2010) hacen énfasis en las tecnologías al expresar que "la colaboración 2.0 incluye un gran número de herramientas informáticas, infraestructuras, servicios y entornos y con frecuencia hace referencia a lo que se denomina como software social”. En relación con lo dicho, para este artículo se considera que no solo la arquitectura de la colaboración 2.0 está conformada por tecnologías y servicios de la Web 2.0, sino que también la conforman: las personas y los procesos organizacionales (véase figura 1).

La arquitectura de colaboración propuesta tiene tres componentes dinámicos e interactivos, a continuación se explica cada uno de ellos:

- Las personas: son los individuos, los participantes de las redes sociales, los expertos, los grupos, las comunidades de práctica, las organizaciones que interactúan, participan y cooperan en un entorno colaborativo; donde se les facilita la transferencia de conocimiento. En el contexto del proceso de transferencia de conocimiento 2.0, las personas que interactúan en un escenario de colaboración son: el emisor, el receptor y los participantes, a saber, los trabajadores del conocimiento (por ejemplo, ingenieros de software, arquitectos, ingenieros, científicos), el equipo de dirección de transferencia de conocimiento, el líder del proceso de transferencia de conocimiento, los directores de las unidades, el director de talento humano, clientes, proveedores, socios, etc.

- Los procesos: son los procesos del negocio y el proceso de transferencia de conocimiento 2.0, sobre los cuales se definen las políticas de colaboración. 


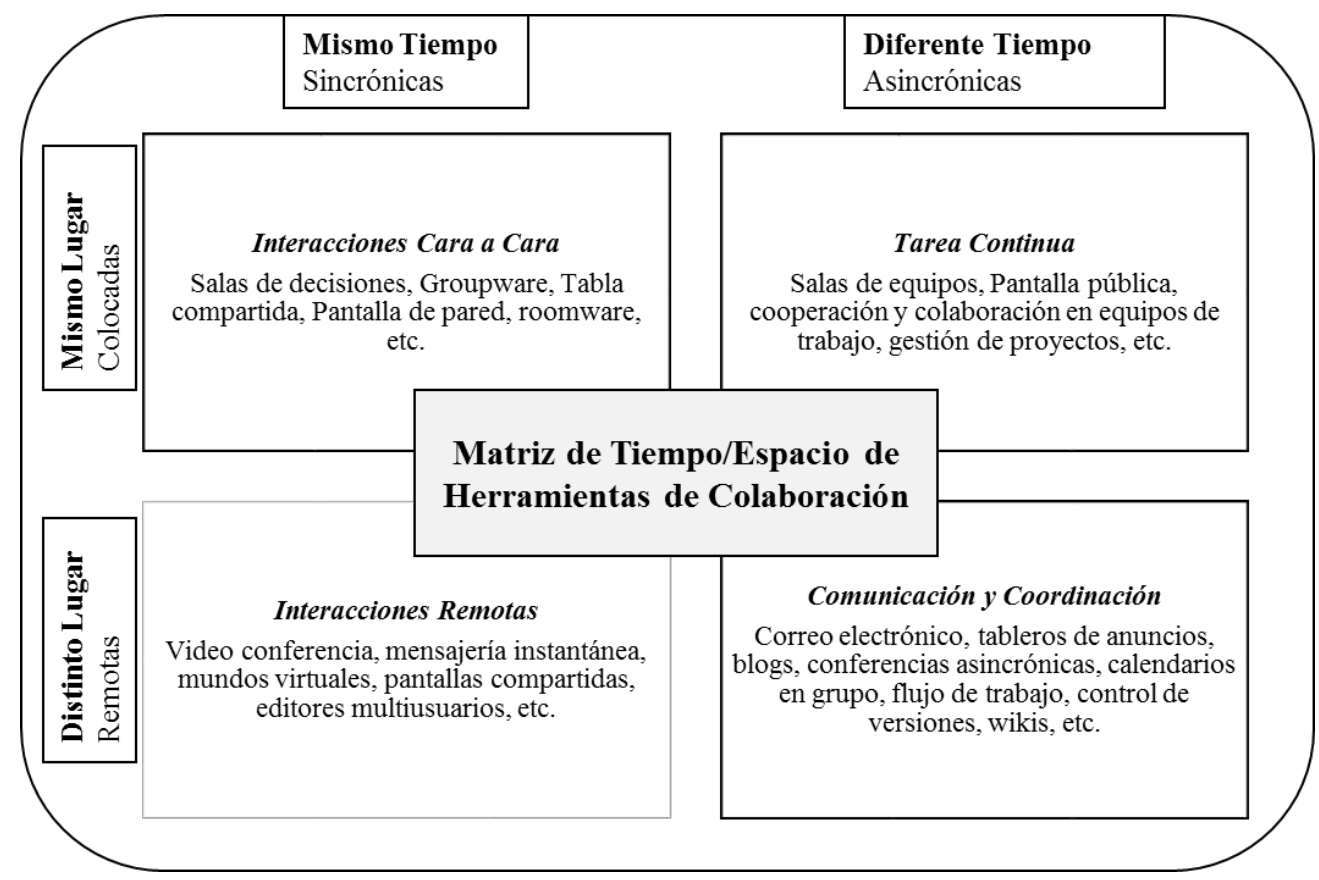

Figura 1. Arquitectura de la colaboración 2.0.

Fuente: [10]

- Tecnologías y servicios de la Web 2.0: son las plataformas tecnológicas de la Web 2.0 que soportan y facilitan las estrategias de colaboración definidas por la organización y el área de TI, estas pueden ser: tecnologías o servicios de la Web 2.0. En este nuevo contexto se plantean retos interesantes para el área de TI de la organización, pues se deben replantear las formas y rutinas de trabajo tradicionales y entrar en las nuevas formas de trabajo y definición de nuevas políticas que el uso de estas tecnologías implican en los modernos escenarios organizacionales.

La arquitectura de colaboración antes descrita alineada con la estructura, la estrategia y la cultura organizacional, establecen nuevos escenarios para el trabajo en red.

Desde esta perspectiva surgen nuevos modelos de colaboración basados en las tecnologías y servicios de la Web 2.0, los cuales están orientadas a resolver las barreras del tiempo y la distancia que pueden ser inhibidores de la colaboración en un contexto organizacional, a continuación se presenta la matriz de colaboración propuesta por los autores Laudon y Laudon (2012), (véase figura 2).

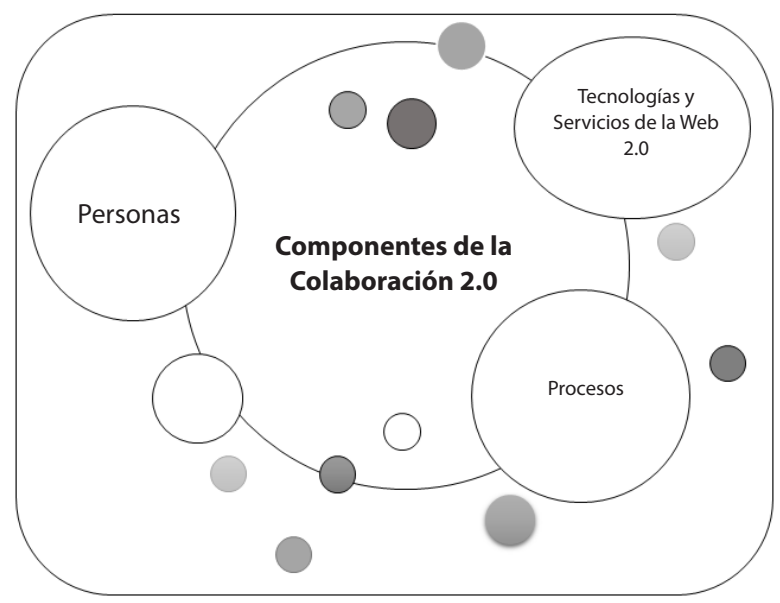

Figura 2. Matriz de tiempo/espacio de herramientas de colaboración.

Fuente: Laudon y Laudon (2012)

La matriz de tiempo y espacio propone una serie de herramientas que pueden ser utilizadas en los contextos organizacionales de acuerdo a cuatro 
componentes combinadas definidas a partir de las características de sincronía y asincronía y de espacio propias de las tecnologías y servicios de la Web 2.0. Sin embargo para lograr esta transición de la colaboración tradicional o colaboración 1.0 a la colaboración 2.0 algunas de los cambios a ser implementados en la organización son los especificados en la tabla 1.

\section{DE LA COLABORACIÓN 1.0 A LA COLABORACIÓN 2.0}

La colaboración 1.0 está basada en sistemas de información propietarios, como son los sistemas de soporte a la toma de decisiones tradicionales
(ERP, CRM, BI, etc.) y la colaboración 2.0 utiliza herramientas del software social y da lugar a nuevas configuraciones tales como las redes sociales o los mundos virtuales (Turban et al., 2010). Este cambio propone la definición y la configuración de nuevos marcos de trabajo en la organización tradicional, y la reta a enfrentar los siguientes cambios, (véase tabla 1)

En resumen, la colaboración 2.0 tiene ventajas significativas sobre la colaboración 1.0 en aspectos como: su bajo costo (algunas de las herramientas son gratuitas), la capacidad de interoperabilidad (debido a su base de código abierto), su marco único participativo (contenido generado por el

Tabla 1. Comparación entre colaboración 1.0 y colaboración 2.0 en un contexto empresarial.

\begin{tabular}{|c|c|c|}
\hline Área & Colaboración 1.0 & Colaboración 2.0 \\
\hline Contexto & Controlada por la empresa & $\begin{array}{l}\text { Generada por los usuarios, flexible y } \\
\text { dinámica }\end{array}$ \\
\hline Facilidad de uso & Puede ser compleja & Muy fácil de usar \\
\hline Costos & Pueden ser muy altos & Muy bajos \\
\hline Plataforma & Propietarias & Software libre, flexible \\
\hline Enfoque & Apoyo a transacciones & Basada en interacciones \\
\hline Nivel de interactividad & Bajo & Alto \\
\hline Naturaleza de la colaboración & $\begin{array}{l}\text { Estructurada, se inicia por la } \\
\text { organización }\end{array}$ & No estructurada, iniciada por los usuarios \\
\hline Aplicaciones adicionales & Creadas por la organización & Pueden ser creada fácilmente por los usuarios \\
\hline $\begin{array}{l}\text { Canales para intercambio de } \\
\text { información }\end{array}$ & Correo electrónico, mensajes de texto & RSS, microblogging (Twitter), blogs, wikis \\
\hline Flujo de información & Estructurado de arriba hacia abajo & No estructurado, de abajo hacia arriba \\
\hline $\begin{array}{l}\text { Contexto de marcado para la búsqueda } \\
\text { de información }\end{array}$ & $\begin{array}{l}\text { Por lo general no se hace, se utiliza el } \\
\text { que brindan los buscadores }\end{array}$ & Creado por los usuarios, folcsonomías \\
\hline Combinación de aplicaciones & Requiere programación compleja & Fácil de hacer gracias a los mashups \\
\hline Contacto con expertos externos & Correo electrónico, contactos propios & $\begin{array}{l}\text { Redes sociales, colaboración masiva, foros, } \\
\text { chats, video conferencia, podcast, etc. }\end{array}$ \\
\hline Ambientes e infraestructura de apoyo & Intranets, extranets. & Redes sociales, intranets y mundos virtuales \\
\hline Flexibilidad & Baja & Alta \\
\hline Software para colaboración & $\begin{array}{l}\text { Estructurado, no puede ser modificado } \\
\text { y debe estar instalado }\end{array}$ & $\begin{array}{l}\text { No estructurado y no necesita estar instala- } \\
\text { do, los usuarios lo proponen. }\end{array}$ \\
\hline
\end{tabular}

Fuente: Turban et al. 2010.

Universidad de Medellín 
usuario, controlado por el usuario y con mayor participación), la capacidad de ser fáciles y rápidas al momento de implementarlas o de crear aplicaciones innovadoras y, por último, las múltiples posibilidades que ofrecen para generar escenarios de colaboración en masa basados en la interactividad.

De las anteriores definiciones y aportes hechos por diferentes autores sobre el emergente concepto de colaboración 2.0 se pueden deducir dos perspectivas o enfoques sobre la misma, uno de ellos es el enfoque tecnológico el cual proponen e impulsan autores como Coleman y Levine (2008) y, otro, es el enfoque socio-técnico el cual proponen autores como McAfee (2009, 2010), Frost y Sullivan (2009), Boughzala (2009) y Boughzala y Dudezert (2012). Sin embargo, para esta investigación interesa la integración adecuada de las dos perspectivas, tanto la organizacional como la tecnológica. En el apartado siguiente se proponen una serie de estrategias que podrán mejorar la colaboración en el modelo de transferencia de conocimiento 2.0.

\section{ESTRATEGIAS DE COLABORACIÓN}

Debido al crecimiento exponencial que ha tenido el uso de los servicios y tecnologías de la Web 2.0 en las organizaciones y los beneficios que se han obtenido de este uso, una de las inquietudes de los gerentes y los directores de TI es definir las estrategias a seguir para su implementación.

Sin bien, al igual que con las tecnologías tradicionales y por las características únicas de los servicios y tecnologías de la Web 2.0, no existe una fórmula mágica que defina cuál es la mejor estrategia para su implementación en la organización, si existen algunas aproximaciones desde la literatura.

Al respecto del uso de la colaboración en la organización, IBM Corporation (2006) expresa que para definir una estrategia integrada de colaboración basada en las tecnologías de la Web 2.0, que favorezca la transferencia de conocimiento en una organización, se deben tener en cuenta los siguientes aspectos:
- Innovación del modelo empresarial: cambiando la manera en que se dirige la organización para conseguir diferenciación competitiva (orientada al cliente, creando nuevos valores), es decir, una organización creadora de conocimiento.

- Colaboración: creando un entorno e infraestructura que fomenten la transferencia de conocimiento e información para la empresa ampliada (empleados, socios y clientes).

- Integración entre TI y el negocio: combinando los conocimientos tecnológicos y los principios de la Web 2.0 con la percepción comercial y de marketing para conseguir los objetivos empresariales, una arquitectura de colaboración.

La clave de estas estrategias es la integración, es decir, la construcción de arquitecturas de colaboración que integren personas, procesos y tecnologías y servicios de la Web 2.0.

En este sentido, al compartir información y conocimiento entre las distintas líneas de negocio, los empleados impulsan de forma natural la transferencia de conocimiento desde su base. Por lo que estas actividades pueden ser una potente fuente de ideas, contactos, redes, flujos de conocimiento y acciones y, por eso, ya muchas organizaciones disfrutan de las ventajas de un personal más conectado, informado y flexible.

\subsection{Tendencias de la Colaboración 2.0}

Como respuesta a la aplicación y el uso cada vez más frecuente de las tecnologías y servicios de la Web 2.0 en las organizaciones, se pueden encontrar estudios recientes realizados por las firmas interesadas en este mercado de las tecnologías. Un ejemplo de estos estudios es el "IBM Global CEO Study 2012”, el cual a partir de sus resultados define las tendencias o futuras aplicaciones de estas tecnologías en la organización y los retos que ello implica. Para comprender mejor los retos 
y objetivos de los directores ejecutivos de hoy, los consultores de IBM se encontraron cara a cara con la muestra más grande conocida de estos ejecutivos. Entre septiembre de 2011 y enero de 2012, 1.709 consejeros delegados, directores generales y altos dirigentes del sector público fueron entrevistados en todo el mundo para comprender mejor sus planes hacia el futuro y los retos de una economía cada vez más conectada.

Algunos de los resultados del estudio de IBM (2012) describen lo siguiente:

Los gerentes consideran las habilidades interpersonales de colaboración (75\%), comunicación (67\%), creatividad (61\%) y flexibilidad (61\%) como factores clave de éxito de los empleados para operar de una manera más compleja, en el medio ambiente interconectado.

Para construir su próxima generación de mano de obra, las organizaciones tienen que reclutar activamente y contratar a los empleados que tienen éxito en el trabajo en equipo, abiertos, basados en entornos. Al mismo tiempo, los líderes deben desarrollar y apoyar las prácticas para ayudar a los empleados a prosperar, prácticas como fomentar el desarrollo de los equipos convencionales, la promoción de técnicas de aprendizaje experiencial y el empoderamiento de la utilización de redes de empleados de alto valor.

La tendencia hacia una mayor colaboración se extiende más allá de la corporación, es decir, hacia las relaciones de asociación. La asociación se encuentra ahora en su punto más alto de todos los tiempos. En 2008, poco más de la mitad de los gerentes entrevistados IBM planeaba asociarse ampliamente. Ahora, más de dos tercios tienen la intención de hacerlo.

"La innovación, junto con sus socios es un gana-gana para ambos”, Peter Voser, consejero delegado de Shell Holandesa Inc., dice. "En Shell, no sólo hay que hacer frente a la energía, también hay que centrarse en los retos, como el agua y los alimentos, ya que todos están relacionados entre sí. Es por eso que impulsar la innovación colaborativa, también a través de las industrias, es muy importante".

En este mismo estudio se encontró que una mayoría (71 \%) de los gerentes ven la tecnología como el factor "número 1" para afectar el futuro de la organización durante los próximos tres años, considerándolo como el agente de cambio más grande de las cambiantes condiciones económicas y de mercado.

En todos los aspectos de la organización, desde las finanzas, competidores y las operaciones, los gerentes están más centrados en ganar un conocimiento más profundo acerca de sus clientes. Siete de cada diez ejecutivos están haciendo inversiones importantes en la capacidad de sus organizaciones para sacar conclusiones significativas de los clientes a partir de datos disponibles.

\section{CONCLUSIONES}

En conclusión, el estudio de IBM Corporation (2006) define que: "las prácticas de colaboración dentro de una organización determinada pueden ser complejas, con procesos, herramientas y requisitos cambiantes y solapados en los distintos roles de la transferencia de conocimiento, segmentos de la empresa y dominios de actividad. Implementar tecnologías de colaboración, como la mensajería instantánea o la videoconferencia para toda la empresa, sin considerar su uso práctico y valor empresarial puede tener un efecto más negativo que positivo. Si la tecnología no está adecuada a las necesidades de los empleados o no se proporciona con suficiente soporte durante la transición, es posible que nunca se logre el objetivo propuesto".

Además, la cultura corporativa y la estructura empresarial se han convertido en aspectos muy importantes para ofrecer un ambiente fértil para la colaboración. En otras palabras, las organizaciones que tienen un proceso de cultura abierto y una estructura descentralizada de toma de decisiones son 
las que tienen entornos óptimos para la adopción y uso de la colaboración entre los empleados y con socios externos.

Por consiguiente, las organizaciones que despliegan herramientas de colaboración pueden aumentar su rendimiento y obtener un retorno positivo de su inversión en la colaboración. En los estudios anteriormente expuestos se muestra que prácticamente cualquier colaboración es buena.

Por último, las organizaciones que sean capaces de hacer inversión en las tecnologías y servicios de la Web 2.0 y estén dispuestas a asegurarse de que su estructura corporativa y el medio ambiente sean propicios para la utilización de las mismas, serán las que luego aprovecharán los beneficios de ellas. Beneficios escalables, que permitirán que los departamentos puedan desarrollar sus capacidades de colaboración de forma inteligente. De esta manera, la colaboración puede convertirse en un activo estratégico de alto desempeño para la organización.

\section{AGRADECIMIENTOS}

Agradecimientos a la Universidad de Medellín por el apoyo dado al proyecto de investigación "Experimentación Modelo de Transferencia de Conocimiento 2.0”, realizado en la empresa de TI Colombiana MVM Ingeniería de Software S.A.

\section{REFERENCIAS}

[1] L. Sáiz, M. Manzanedo, R. del Olmo y R. Alcalde, «Business Management 2.0. Since the hierarchical structure to Knowledge Networks. Model CIACO_RED,» de 5th International Conference on Industrial Engineering and Industrial Management, XV Congreso de Ingenieria de Organización, Cartagena, 2011.

[2] Frost \& Sullivan, «Meetings Around the World II: Charting the Course of Advanced Collaboration,» Verizon and Cisco, 2009.

[3] D. Coleman y S. Levine, Collaboration 2.0: technology and best practices for successful collaboration in a Web 2.0 world., Happy about info publishing company, 2008.

[4] I. Boughzala y A. Dudezert, Knowledge Management 2.0: Organizational Models and Strategies, United States of America: IGI Global, 2012.

[5] E. Turban, . T.-P. Liang y. S. P. J.Wu, «A Framework for Adopting Collaboration 2.0 Tools for Virtual Group Decision Making,» Springer Science+Business Media B.V., 2010.

[6] K. Laudon y J. Laudon, Sistemas de Información Gerencial, México: PEARSON, 2012.

[7] T. O’Reilly, «What Is Web 2.0: Design Patterns and Business Models for the Next Generation of Software," 2005a. [En línea]. Available: http://www.oreilly.com/ pub/a/oreilly/tim/news/2005/09/30/what-is-web-20. html. [Último acceso: 2012].

[8] IBM Corporation, «Uso de la colaboración para fomentar la innovación en su organización,» IBM Global Services, 2006.

[9] IBM Global Study, «IBM CEO Study: Command \& Control Meets Collaboration,» 2012. [En línea]. Available: http://www-03.ibm.com/press/us/en/pressrelease/37793.wss. [Último acceso: 2012].

[10] L. Giraldo, «Modelo de transferencia de conocimiento 2.0 - COOPIN,» Universidad Pontificia de Salamanca, Madrid, 2013.

[11] A. McAfee, «Andrew McAfee's Blog,» Mayo 2006. [En línea]. Available: http://andrewmcafee.org/2006/05/ enterprise_20_version_20/. [Último acceso: Oct 2012].

[12] P. Anderson, «What is Web 2.0?,» JISC Technology and Standards Watch, 2007.

[13] E. Brynjolfsson y A. McAfee, «Beyond enterprise 2.0.," MIT sloanmanagement review spring, p. 50-55, 2007.

[14] J. Bernoff y C. Li, «Harnessing the power of theOh-SoSocial web,» MIT Sloan manage, p. 36-42, 2008. 